\title{
Adsorption Behavior of Glucosamine Based Pyrimidine-Fused Heterocycles as Green Corrosion Inhibitors for Mild Steel: Experimental and Theoretical Studies
}

\author{
Chandrabhan Verma ${ }^{a}$, L.O. Olasunkanmi ${ }^{b, c}$, Eno E. Ebenso ${ }^{b, c}$, \\ M.A. Quraishi ${ }^{\text {a, }}{ }^{*}$ and I.B. Obot ${ }^{\mathrm{d}}$ \\ ${ }^{a}$ Department of Chemistry, Indian Institute of Technology, Banaras Hindu University, Varanasi \\ 221005, India \\ ${ }^{b}$ Department of Chemistry, School of Mathematical \& Physical Sciences, Faculty of Agriculture, \\ Science and Technology, North-West University (Mafikeng Campus), Private Bag X2046, \\ Mmabatho 2735, South Africa \\ ${ }^{c}$ Material Science Innovation \& Modelling (MaSIM) Research Focus Area, Faculty of \\ Agriculture, Science and Technology, North-West University (Mafikeng Campus), Private Bag \\ X2046, Mmabatho 2735, South Africa \\ ${ }^{d}$ Center of Research Excellence in Corrosion, Research Institute, King Fahd University of \\ Petroleum and Minerals, Dhahran 31261, Saudi Arabia
}




\section{SUPPORTING INFORMATION:}

5-phenyl-10-((3R,5S,6R)-2,4,5-trihydroxy-6-(hydroxymethyl)tetrahydro-2H-pyran-3-yl)-9,10dihydropyrido[2,3-d:6,5-d']dipyrimidine-2,4,6,8(1H,3H,5H,7H)-tetraone $\quad$ (CARB-1): Mol. Formula: $\mathrm{C}_{21} \mathrm{H}_{21} \mathrm{~N}_{5} \mathrm{O}_{9}$; Mol. wt. 487.42; Yellow crystals; Yield =79\%; mp 198-200 ${ }^{\circ} \mathrm{C}$; FT-IR $\left(\mathrm{KBr}, \mathrm{cm}^{-1}\right): 3682,3476,3256,3052,2986,2859,2572,2384,1748,1635,1578,1448,1426$, 1369, 1254, 1232, 1124, 1043, 1008, 956, 876, 834, 722, 646; 1H-NMR (500MHz, DMSOd6/TMS) $\delta(\mathrm{ppm}): 2.500(\mathrm{~S}, 3 \mathrm{H}), 3.344(\mathrm{t}, 1 \mathrm{H}), 6.610-6.840(\mathrm{~m}, 5 \mathrm{H}), 7.067-7.110(\mathrm{~m}, 5 \mathrm{H})$, 7.332-7.474 (m, 4H), 11.116-11.177 (q, 1H), 11.202-11.281 (q, 1H)

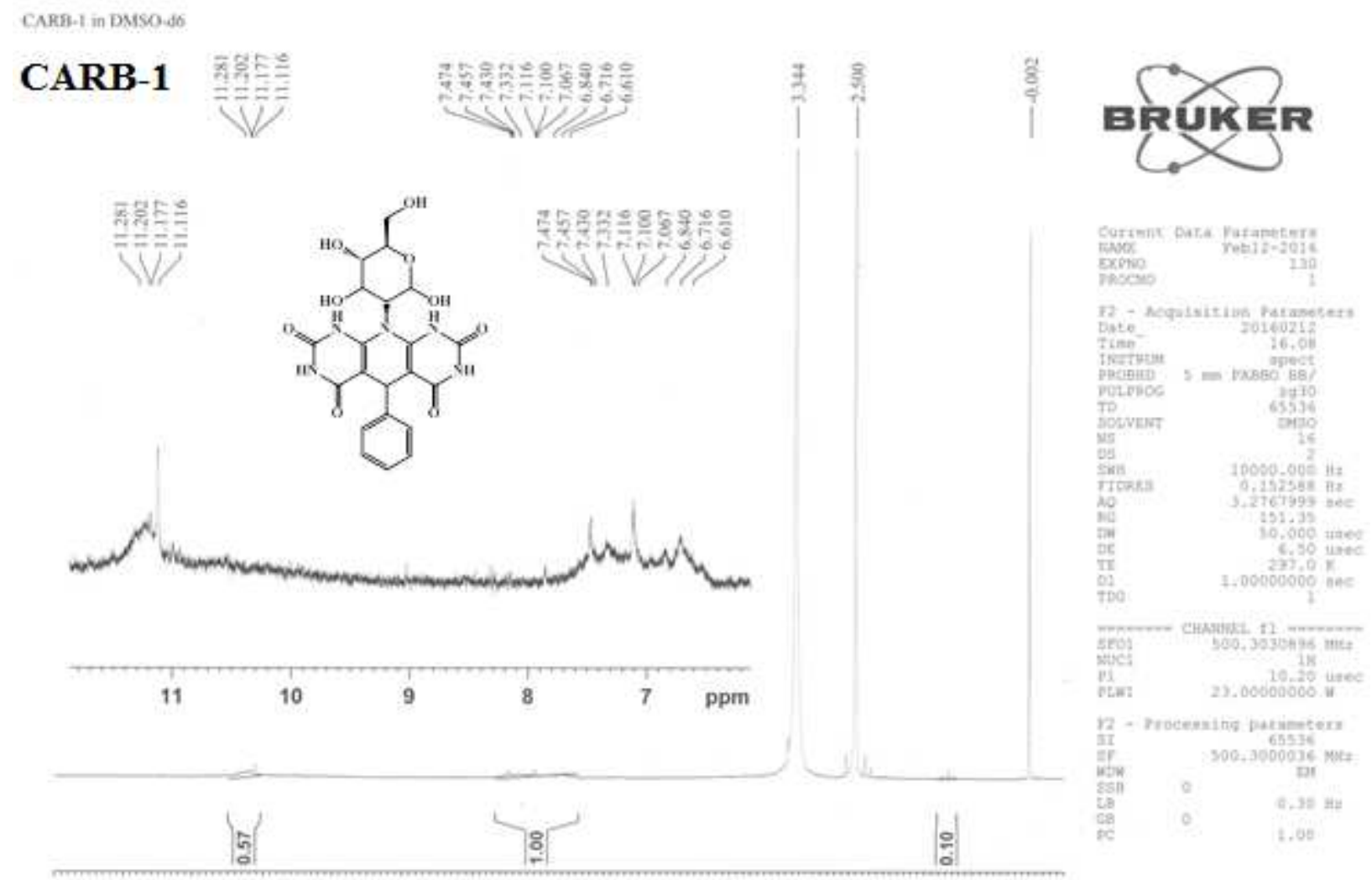


5-(4-nitrophenyl)-10-((3R,5S,6R)-2,4,5-trihydroxy-6-(hydroxymethyl)tetrahydro-2H-pyran-3yl)-9,10-dihydropyrido[2,3-d:6,5-d']dipyrimidine-2,4,6,8(1H,3H,5H,7H)-tetraone (CARB-2): Mol. Formula: $\mathrm{C}_{21} \mathrm{H}_{20} \mathrm{~N}_{6} \mathrm{O}_{11}$; Mol. wt. 532.41; Yellow crystals; Yield $=84 \%$; mp 198-200 ${ }^{\circ} \mathrm{C}$; FT-IR $\left(\mathrm{KBr}, \mathrm{cm}^{-1}\right)$ : 3648, 3456, 3286, 3028, 2984, 2806, 2568, 2334, 1689, 1622, 1542, 1478, 1362, 1287, 1245, 1167, 1079, 1017, 884, 778, 743, 654; 1H-NMR (500MHz, DMSO-d6/TMS) $\delta(\mathrm{ppm}): 1.145(\mathrm{~S}, 1 \mathrm{~h}), 2.500(\mathrm{~S}, 1 \mathrm{H}), 3.350(\mathrm{t}, 1 \mathrm{H}), 6.582-6.968(\mathrm{~m}, 5 \mathrm{H}), 7.070-7.779(\mathrm{~m}, 5 \mathrm{H})$, 7.779-7.956 (m, 5H), 8.007-8.637 (m, 1H), $11.004(\mathrm{~S}, 1 \mathrm{H}), 11.114-11.333(\mathrm{~m}, 1 \mathrm{H})$.

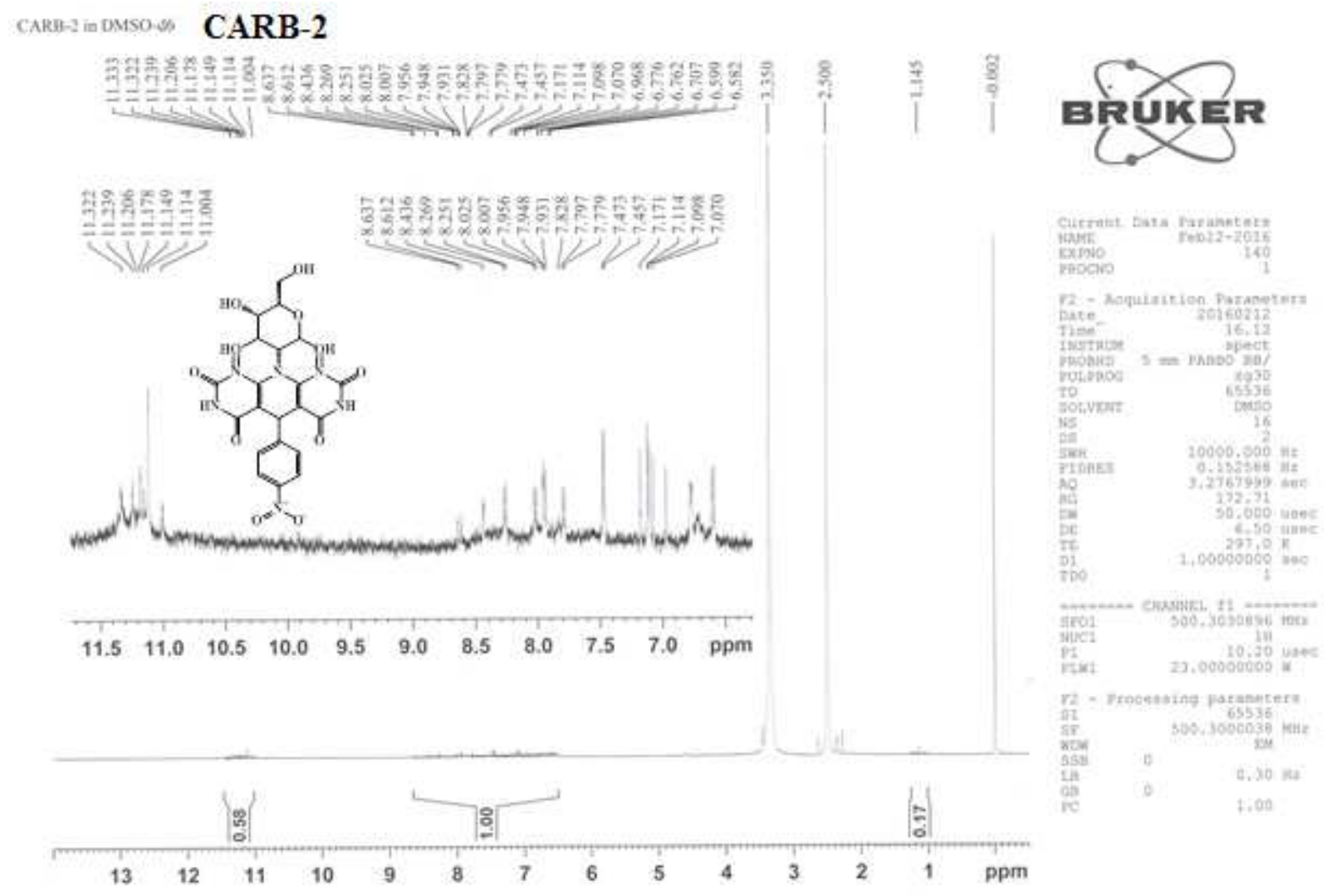


5-(p-tolyl)-10-((3R,5S,6R)-2,4,5-trihydroxy-6-(hydroxymethyl)tetrahydro-2H-pyran-3-yl)-9,10dihydropyrido[2,3-d:6,5-d']dipyrimidine-2,4,6,8(1H,3H,5H,7H)-tetraone (CARB-3): Mol. Formula: $\mathrm{C}_{22} \mathrm{H}_{23} \mathrm{~N}_{5} \mathrm{O}_{9}$; Mol. wt. 501.44; Yellow crystals; Yield $=79 \%$; mp $251^{\circ} \mathrm{C}$; FT-IR $(\mathrm{KBr}$, $\left.\mathrm{cm}^{-1}\right): 3778,3456,3284,3224,2958,2854,2364,1776,1688,1548,1536,1452,1428,1362$, 1192, 1132, 1018, 953, 846, 673; 1H-NMR (500MHz, DMSO-d6/TMS) $\delta(\mathrm{ppm}): 1.146(\mathrm{~S}, 1 \mathrm{H})$, $1.236(\mathrm{~S}, 1 \mathrm{H}), 2.500(\mathrm{~S}, 3 \mathrm{H}), 3.340(\mathrm{t}, 1 \mathrm{H}), 3.724-3.888(\mathrm{~m}, 4 \mathrm{H}), 6.917-6.980(\mathrm{~m}, 3 \mathrm{H}), 7.054-$ $7.115(\mathrm{~m}, 3 \mathrm{H}), 10.793-10.929(\mathrm{~d}, 2 \mathrm{H}), 11.114-11.300(\mathrm{~m}, 1 \mathrm{H}), 11.314-11.459(\mathrm{~m}, 1 \mathrm{H})$.

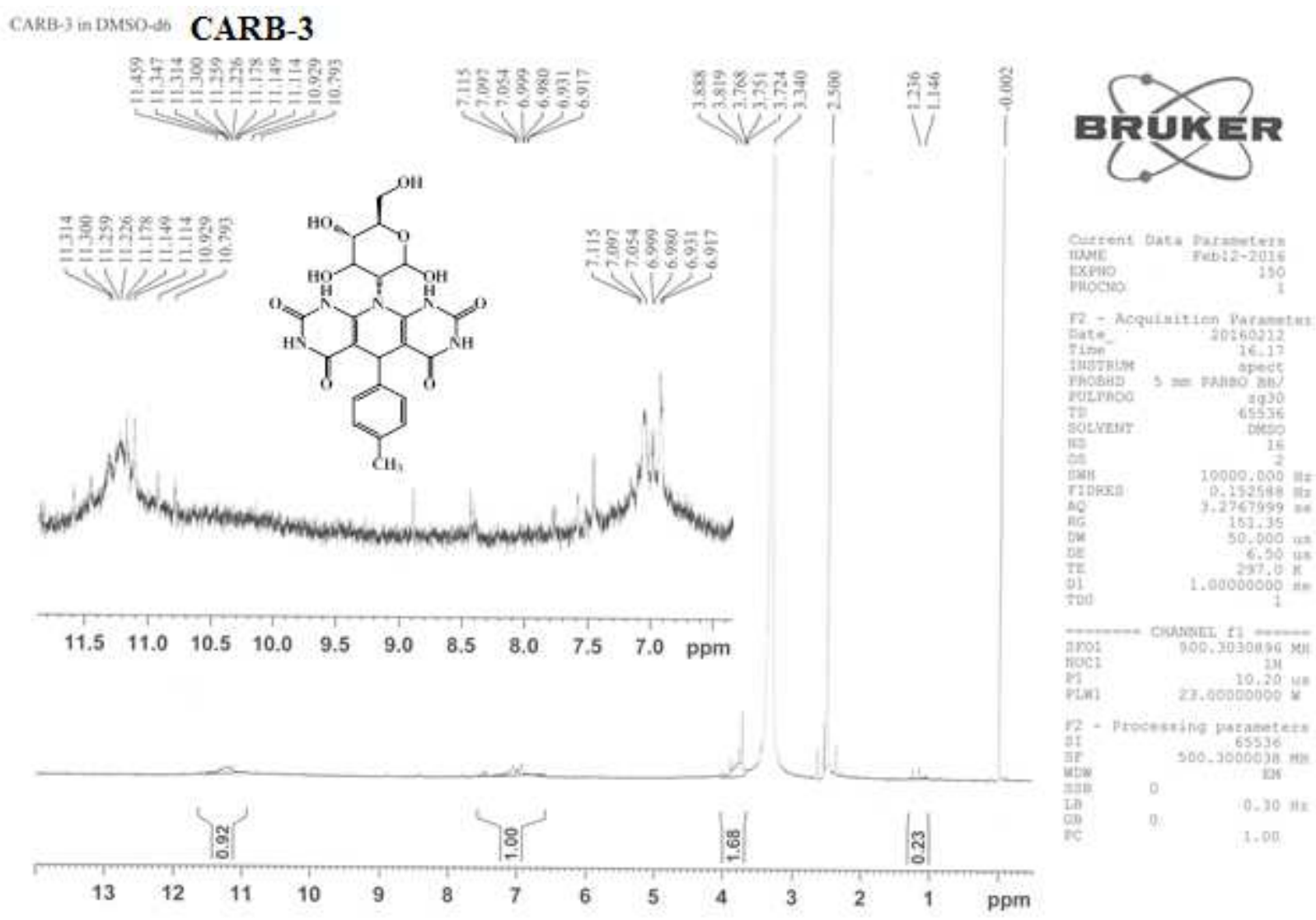


5-(4-hydroxyphenyl)-10-((3R,5S,6R)-2,4,5-trihydroxy-6-(hydroxymethyl)tetrahydro-2H-pyran3-yl)-9,10-dihydropyrido[2,3-d:6,5-d']dipyrimidine-2,4,6,8(1H,3H,5H,7H)-tetraone (CARB-4): Mol. Formula: $\mathrm{C}_{21} \mathrm{H}_{21} \mathrm{~N}_{5} \mathrm{O}_{10}$; Mol. wt. 503.41; Yellow crystals; Yield $=78 \%$; mp 208-210 ${ }^{\circ}$; FT-IR $\left(\mathrm{KBr}, \mathrm{cm}^{-1}\right)$ : 3662, 3458, 3286, 3059, 2986, 2867, 2324, 1992, 1746, 1568, 1514, 1449, 1312, 1276, 1196, 1132, 1017, 998, 926, 882, 837, 742, 623; 1H-NMR (500MHz, DMSOd6/TMS) $\delta(\mathrm{ppm}): 1.146-1.234(\mathrm{~d}, 1 \mathrm{H}), 2.500(\mathrm{~S}, 1 \mathrm{H}), 3.337(\mathrm{t}, 1 \mathrm{H}), 3.458(\mathrm{t}, 1 \mathrm{H}), 6.785-6.956$ $(\mathrm{m}, 5 \mathrm{H}), 7.062-7.170(\mathrm{~m}, 5 \mathrm{H}), 11.221-11.207$ (q, 1H), 11.177-11.114 (q, 1H)

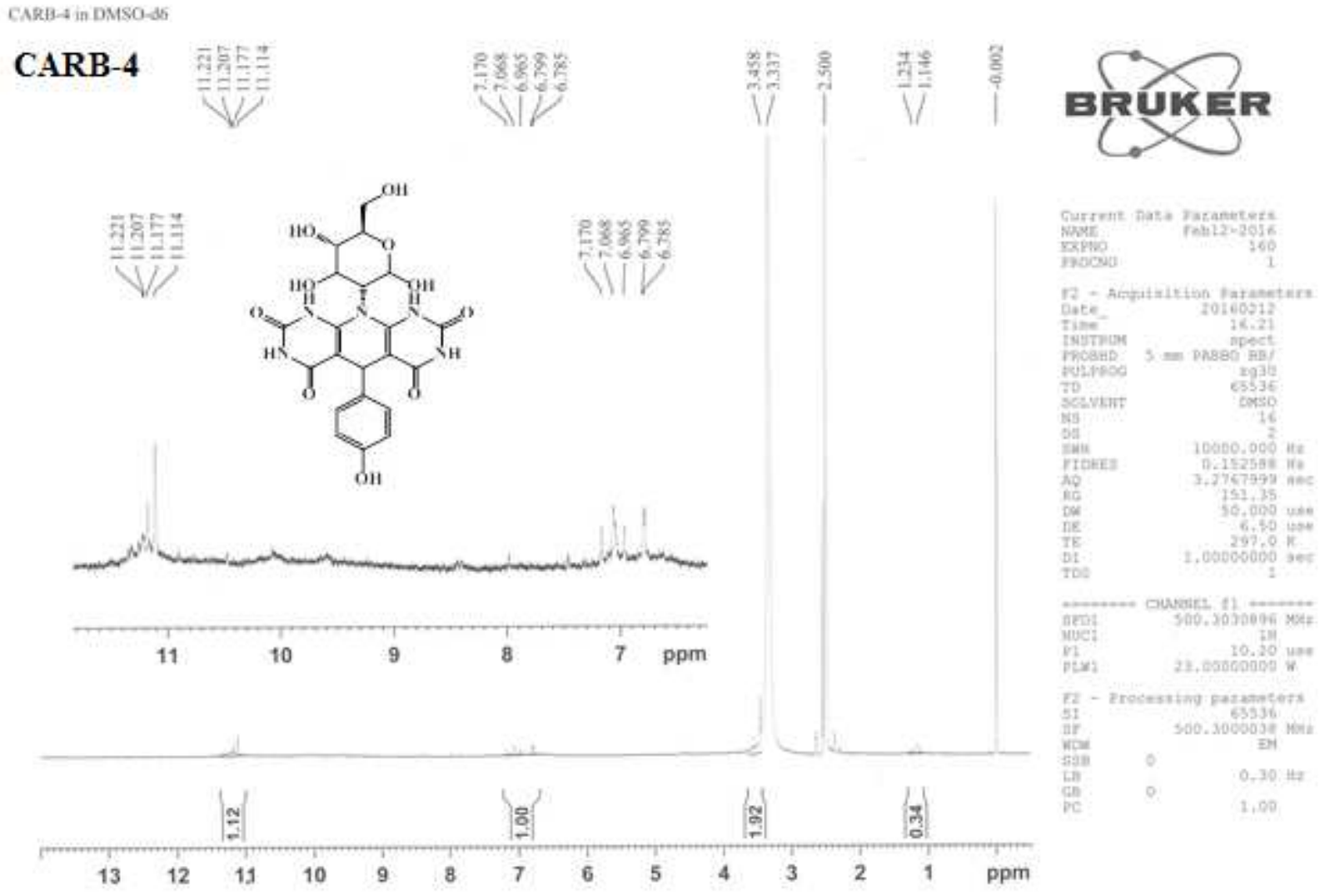




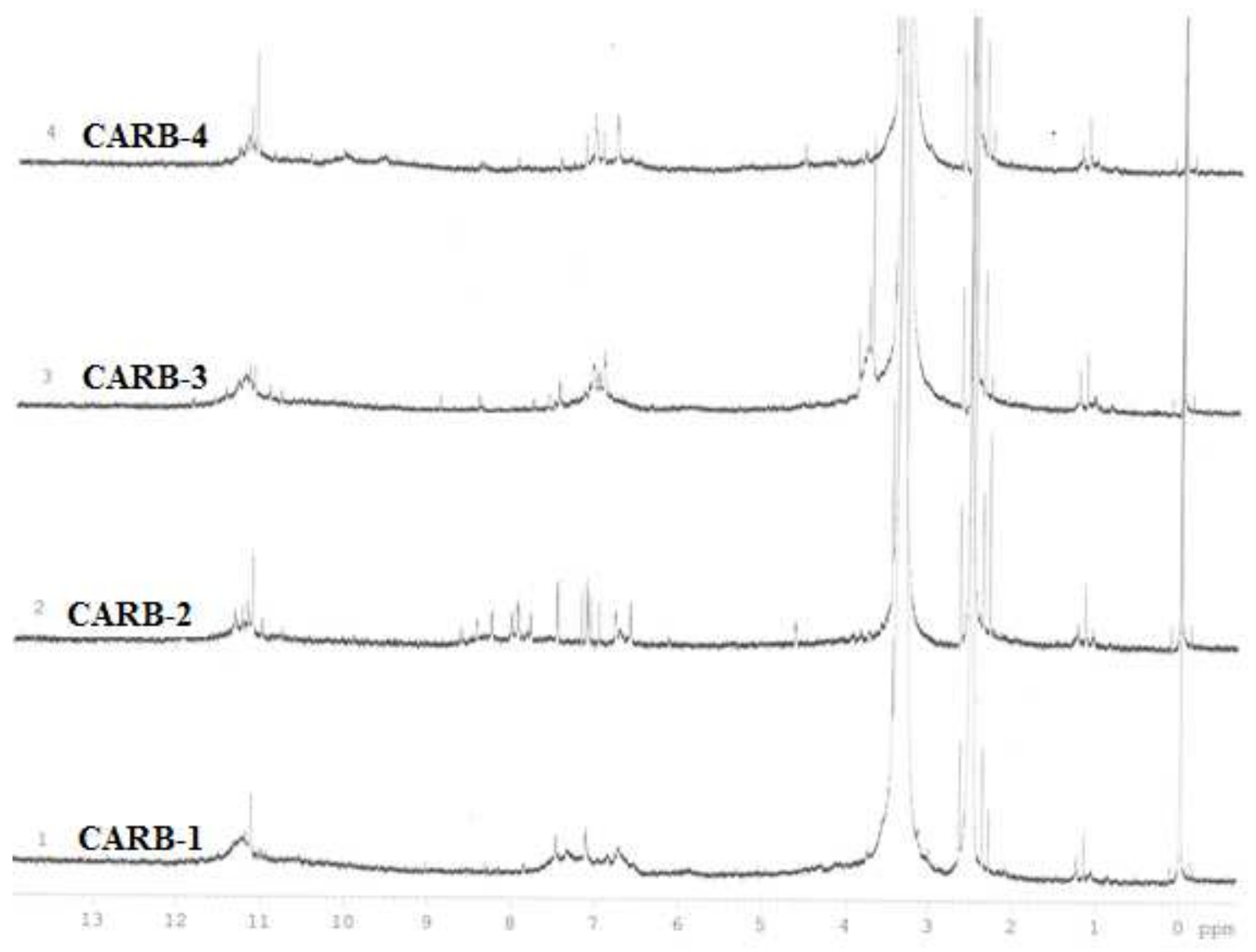

Figure S1: 1H NMR spectra of glucosamine based substituted pyrimidine-fused heterocycles (CARBs) used in the present study 

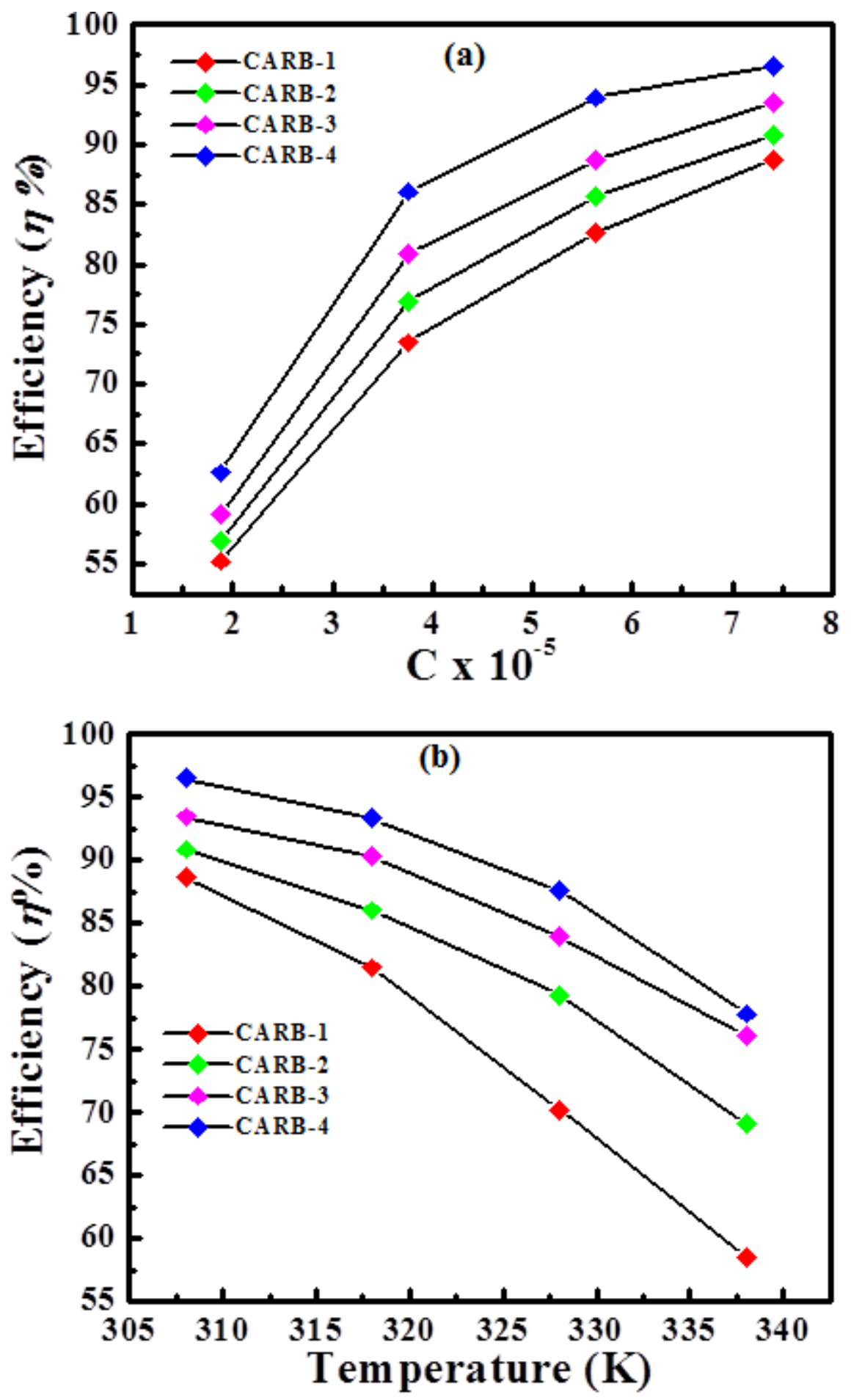

Figure S2: (a) Variation of inhibition efficiency with CARBs concentration

(b) Variation of inhibition efficiency with solution temperature 


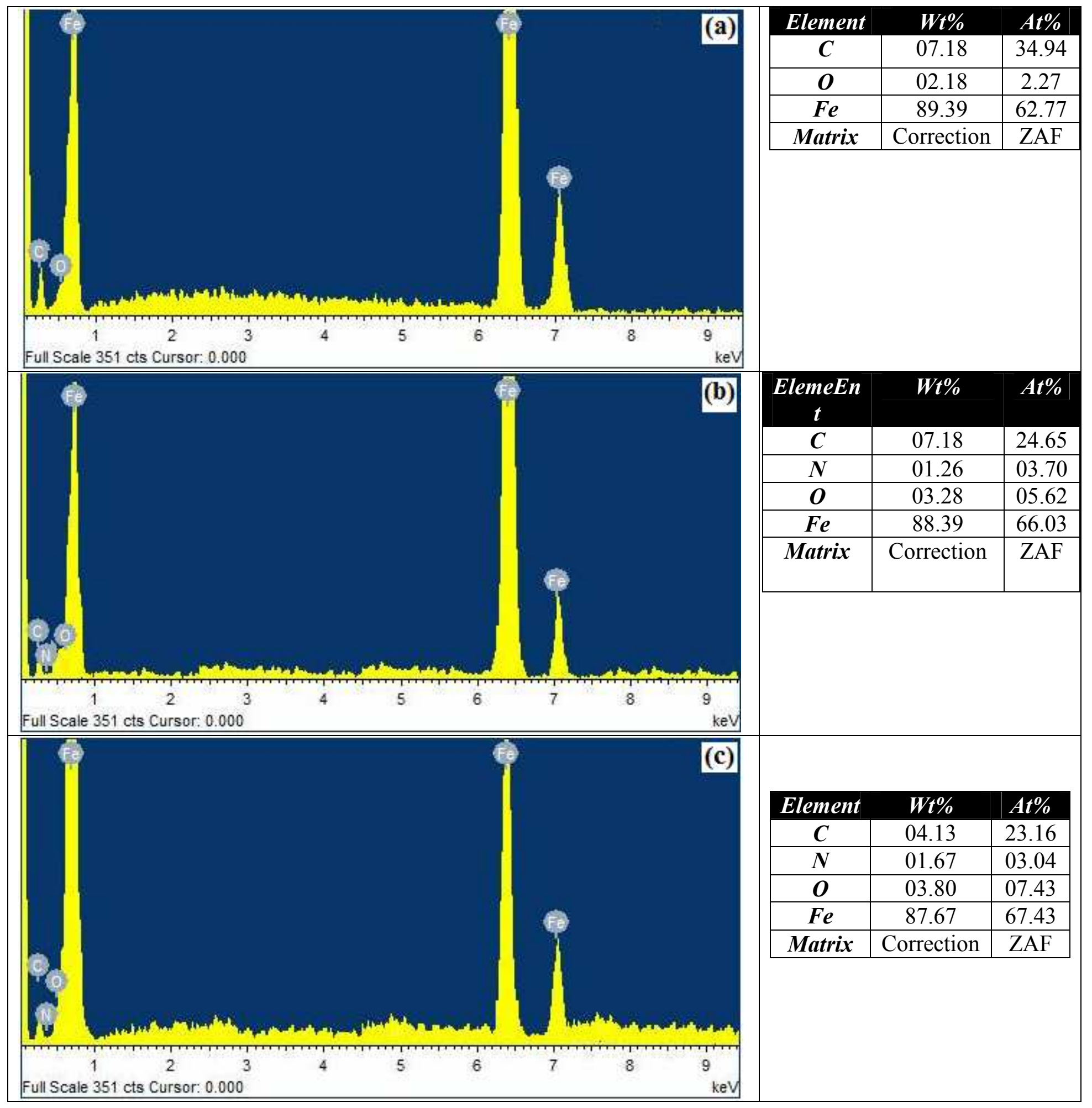




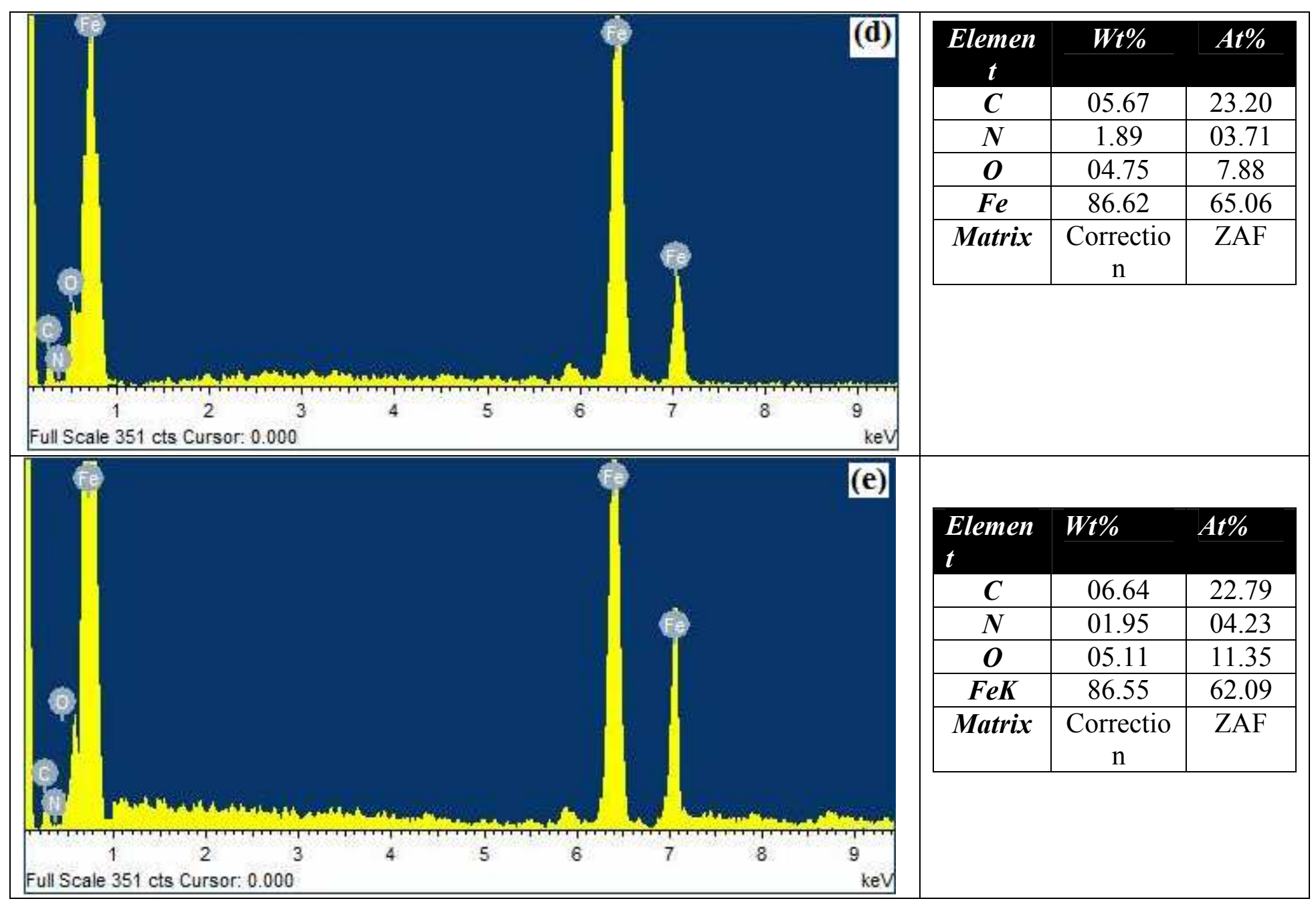

Figure S3: EDX spectra and corresponding elemental composition of mild steel surface obtained for (a) in absence of inhibitors and presence of (b) CARB-1, (c) CARB-2, (d) CARB-3 and (e) CARB-4 
Table S1: Slopes, intercepts, and regression coefficients $\left(R^{2}\right)$ for Langmuir and Temkin adsorption isotherms plots for investigated inhibitors

\begin{tabular}{ccccccccccc}
\hline Inhibito & \multicolumn{3}{c}{ Langmuir adsorption } & \multicolumn{3}{c}{ Temkin adsorption } & \multicolumn{3}{c}{ Freundluich isotherm } \\
r & \multicolumn{3}{c}{ isotherm } & \multicolumn{3}{c}{ isotherm } \\
& \multicolumn{3}{c}{} & & \multicolumn{3}{c}{} & & & \\
& slope & Intercept & $\left(R^{2}\right)$ & slope & Intercept & $\left(R^{2}\right)$ & slope & Intercept & $\left(R^{2}\right)$ \\
CARB-1 & 1.3128 & 6.2123 & 0.9929 & 0.5584 & 3.1620 & 0.9923 & 0.345 & 1.3798 & 0.9873 \\
CARB-2 & 1.4375 & 6.8307 & 0.9964 & 0.5685 & 3.2666 & 0.9901 & 0.3413 & 1.3785 & 0.9754 \\
CARB-3 & 1.6256 & 7.7500 & 0.9961 & 0.5757 & 3.3277 & 0.9793 & 0.3344 & 1.3645 & 0.9615 \\
CARB-4 & 2.0338 & 9.7207 & 0.9981 & 0.5780 & 3.3800 & 0.9556 & 0.3203 & 1.3255 & 0.937 \\
\hline
\end{tabular}

Table S2: Values of slope, intercept, correlation coefficient and phase angle calculated form Bode plots of the in absence and presence of studied compounds

\begin{tabular}{lllll}
\hline inhibitors & $\begin{array}{l}\text { Slope } \\
\text { Decades/Decade }\end{array}$ & $\begin{array}{l}\text { Intercept } \\
\text { kohm }\end{array}$ & $\begin{array}{l}\text { Correlation } \\
\text { coefficient }\end{array}$ & Phase angle \\
\hline Blank & -0.4805 & 0.0926 & -0.99529 & -41.3 \\
CARB-1 & -0.7910 & 0.5976 & -0.99984 & -66.3 \\
CARB-2 & -0.8136 & 1.581 & -0.99944 & -68.9 \\
CARB-3 & -0.8292 & 1.053 & -0.99988 & -72.2 \\
CARB-4 & -0.8163 & 2.767 & -0.99893 & -73.6 \\
\hline
\end{tabular}

